\title{
A Review on Deep Image Contrast Enhancement
}

\author{
Puspad Kumar Sharma \\ M. Tech. Scholar \\ Department of CSE \\ National Institute for \\ Interdisciplinary Science and \\ Technology, Bhopal, India \\ puspad72@gmail.com
}

\author{
Nitesh Gupta \\ Assistant Professor \\ Department of CSE \\ National Institute for \\ Interdisciplinary Science and \\ Technology, Bhopal, India \\ 9.nitesh@gmail.com
}

\author{
Anurag Shrivastava \\ Associate Professor \\ Department of CSE \\ National Institute for \\ Interdisciplinary Science and \\ Technology,Bhopal, India \\ anurag.shri08@gmail.com
}

\begin{abstract}
In image processing applications, one of the main preprocessing phases is image enhancement that is used to produce high quality image or enhanced image than the original input image. These enhanced images can be used in many applications such as remote sensing applications, geo-satellite images, etc. The quality of an image is affected due to several conditions such as by poor illumination, atmospheric condition, wrong lens aperture setting of the camera, noise, etc [2]. So, such degraded/low exposure images are needed to be enhanced by increasing the brightness as well as its contrast and this can be possible by the method of image enhancement. In this research work different image enhancement techniques are discussed and reviewed with their results. The aim of this study is to determine the application of deep learning approaches that have been used for image enhancement. Deep learning is a machine learning approach which is currently revolutionizing a number of disciplines including image processing and computer vision. This paper will attempt to apply deep learning to image filtering, specifically low-light image enhancement. The review given in this paper is quite efficient for future researchers to overcome problems that helps in designing efficient algorithm which enhances quality of the image.
\end{abstract}

Keywords - Image enhancement, Image quality, Deep learning approaches, Digital image processing.

\section{INTRODUCTION}

In the last decade, we saw a surge in multimedia content generated across the world. With the arrival of digital equipment like cameras, camcorders, etc., it became feasible for a large section of the world's population to generate such content. These devices have become increasingly popular, especially to improve their performance, their gradual erosion of prices and their integration into mobile phones. In fact, with the increasing mobility of these devices via mobile phones, combined with advances in low-cost storage, it was possible to capture multimedia content in the form of images and videos in an instant.

The large volume of multimedia content generated by the population has given us the opportunity to label, classify and make it searchable [1]. This scenario has become even more important as the content can be downloaded from the Internet, to which millions of people can access. Man-directed tasks, such as commenting on content, to identify people in a stream of surveillance cameras have become difficult with increasing content [2].

In real time applications an enhancement technique should be capable of enhancing real color images in lesser time with lesser computational cost by reducing:

i. the effect of haze or fog

ii. poor or uneven illumination effect on an image

iii. noise introduced in an image

This research review work focus on various color image enhancement techniques that improves the contrast of real time images.

Requirements of real time image enhancement techniques [2] are:

i. It should be adaptive in nature i.e. should be able to enhance any type of images for a specific application.

ii. Should enhance an image in less processing time.

iii. It should utilize less computational resources.

\section{IMAGE ENHANCEMENT TECHNIQUES}

In digital image processing field one of the preprocessing technique is image enhancement which generates improved quality of the image out of the distorted or low enhanced image which shows its efficiency for applications in many areas and human interactions The image enhancement techniques are divided into two types, one is spatial techniques and other is frequency domain image enhancement [1]. In spatial domain image enhancement methodology, the pixels of an image enhance themselves directly in order to improve the image quality. Whereas in frequency domain image enhancement techniques, frequency transformation is performed on the pixel intensity values for their enhancement. 


\section{A. Gray Level Transformation}

One of the simplest enhancement techniques is grayscale transformation. In this technique, input image is converted into grayscale and processing are performed on pixel directly. These are represented as following:

$$
\mathrm{s}=\mathrm{T}(\mathrm{r})
$$

where,

$\mathrm{T}=$ transformation function

$\mathrm{r}=$ input pixel value

$\mathrm{s}=$ transformed pixel value

In general, image contrast is enhanced with grayscale transformation. This is performed by adjusting the gray level values of the pixels which is calculated by finding difference between minimum and maximum pixel values in a region of image. Linear, logarithmic, and power-law are most commonly used transformation function in this technique.

Advantages of Thresholding transformations are :

i. Thresholding transformations are particularly useful for segmentation in which we want to isolate an object of interest from a background.

ii. Simple to use and less complex than others.

\section{B. Contrast Stretching}

It is seen that low contrast of an image is caused by several factors such as low lightning condition, low illumination of camera, background light or atmospheric conditions. Contrast stretching or expansion is one of the simplest methodology. By increasing the gray level of an image dynamically, contrast can be stretched [6]. The stretching of contrast refers to stretch pixel values between lower and upper threshold values of the pixels. The stretching is represented as:

$$
\mathrm{I}_{0}(\mathrm{x}, \mathrm{y})=\mathrm{f}(\mathrm{I}(\mathrm{x}, \mathrm{y}))
$$

Where,

$\mathrm{I}(\mathrm{x}, \mathrm{y})=$ input image

$\mathrm{I}_{0}(\mathrm{x}, \mathrm{y})=$ output enhanced image

The transformation function $\mathrm{T}$ is given by

$$
\mathrm{s}=\mathrm{T}(\mathrm{r})
$$

Advantages of Contrast Stretching are:

i. Contrast Stretch is the simplest contrast stretch algorithm that stretches the pixel values of a low-contrast image or high-contrast image by extending the dynamic range across the whole image spectrum.

\section{Histogram Processing}

Histogram equalization is one of the main pixel intensity transformation functions which is based on intensity histograms information extracted from an image.

With $\mathrm{L}$ total possible intensity levels in the range $[0, \mathrm{G}]$, the histogram of a digital image is defined as the discrete function:

$$
\mathrm{h}\left(\mathrm{r}_{\mathrm{k}}\right)=\mathrm{n}_{\mathrm{k}}
$$

where,

$\mathrm{r}_{\mathrm{k}}=\mathrm{kth}$ intensity level in the interval $[0, \mathrm{G}]$

$\mathrm{n}_{\mathrm{k}}=$ number of pixels with intensity level $\mathrm{r}_{\mathrm{k}}$ in an image

Advantages of histogram equalization are:

i. This technique is very simple.

ii. Only the global histogram equalization can be done completely automatically.

\section{Smoothing Filters}

By transformation of edges and intensity in the grayscale, high frequency can be achieved. Therefore, by attenuating a specified range of high-frequency components, smoothing or blurring is generated in an image. This can be performed by low pass filter and thus enhancement is achieved. Function of low pass filter is to attenuate high frequencies by keeping low frequencies unchanged. A spatial area is created by blocking the high frequencies range by using filters. Generally for this purpose three low pass filter are used such as ideal low-pass filter, Butterworth low-pass filter and Gauss low-pass filter.

\section{E. Sharpening Filters}

One of the available filters is Sharpening filters which is used where there is need for sharpening edges. This is done by finding differences between adjacent light and dark region in an image. In spatial domain, contour enhancement or boundary detection is achieved by high-pass filter because they contain many high frequencies [8]. In an image, the continuous gray areas contains mainly low frequencies. That is reason they are seems to be suppressed. By inverse transform over low pass filter, corresponding high-pass filter can be obtained. The transfer function of the low pass filter and the high pass filter can be correlated as follows:

$$
H_{h p}(u, v)=1-H_{l p}(u, v)
$$

Where,

$\mathrm{H}_{\mathrm{hp}}(\mathrm{u}, \mathrm{v})=$ transfer function of highpass filter

$\mathrm{H}_{\mathrm{lp}}(\mathrm{u}, \mathrm{v})=$ transfer function of lowpass filter 


\section{RELATED WORK}

Low-level light enhancement is a common aim in the field of image processing. Images captured in lowlight, or due to a low-exposure, suffer from low contrast and brightness, which both degrades the visual quality of the image and can hinder further image processing algorithms (e.g. object detection [2]).
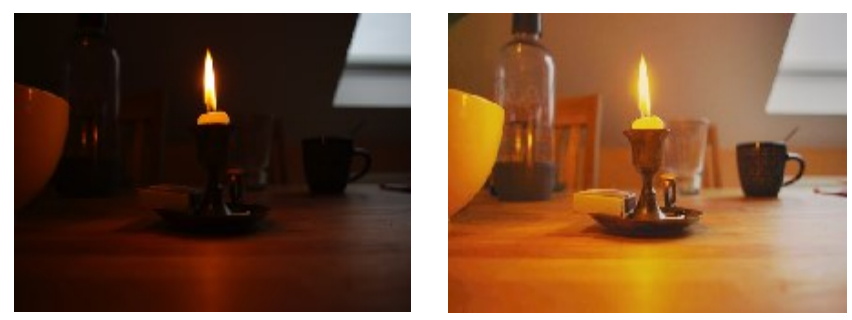

Figure 1: Low-level light images vs filtered images

There have been many attempts to correct for low-level light noise, from regular histogram-based methods as discussed by Shen et al. in [3] to adaptive gamma correction in [2] and illumination map estimation as discussed by Guo et al. in [4].

Figure 1 shows two examples of images captured in low-light, compared to their filtered alternatives [5].

Low light images have been enhanced successfully using histogram and saturation based methods, for example Lim et al. [6] and Yoo et al. in [7].

Ramiz and Quazi [8] proposed an effective image quality enhancement technique. In this enhancement technique frequency and spatial domain, both, are processed and thus this algorithm is termed as hybrid technology. The performance parameter for simulation of this algorithm are Mean Square Error (MSE) and Peak Signal to noise ratio (PSNR). The experimental results shows enhanced PSNR value and low MSE.

While these methods have generated promising outputs, the running time for each process can be large. With deep learning, the network is trained (which takes significant time) and produces a filter which can be run quickly and with minimal processing, for example on a smartphone. Deep learning has been used to filter low-light images, with successful results [9]. Due to the successes of using deep learning for low-level noise reduction, this paper will attempt to analyses different methods of applying deep learning to image filtering.

\section{DEEP LEARNING IN IMAGE PROCESSING}

In image processing, deep learning is often used for image classification but has also been used for image filtering. One of the biggest issues in applying deep learning to image processing is how to input the image data into the neural network. A simple approach would be to have each pixel being an input to the neural network. However, with a standard www.ijoscience.com photograph size of 5 "x7", a typical resolution is $630 \times 450$ pixels.

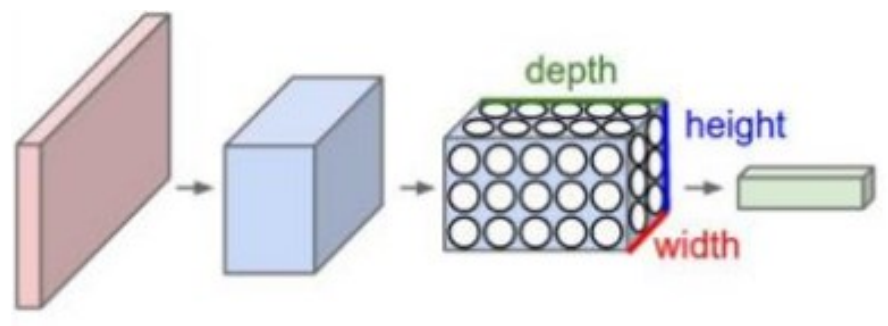

Figure 2: Convolutional Neural Networks [10]

If the image is in RGB form as opposed to grayscale, this value gets multiplied by 3 . This requires a neural network with 850,500 input notes, which can rapidly increase the size of the network if multiple hidden layers are used, dramatically increasing the required computation time for the network to be trained.

To combat this issue, convolutional neural networks (CNNs) were created and will be used as the foundation of this project. These are a type of neural network which is designed specifically to be used with images, and differ slightly from traditional neural network structure. Each layer in a $\mathrm{CNN}$ is a 3D structure of nodes, as opposed to a 1D structure in regular neural networks. This can be seen more clearly in Figure 2.

Another key property of CNNs is that they are not fully connected [10], where every node in a layer is connected to every node in the previous layer.

There are four main elements to a $\mathrm{CNN}$ :

- Convolutional layer

- $\quad$ Rectified Linear Unit

- Pooling layer

- Fully connected layer

\section{A. Convolutional Layer}

The convolutional layer is a layer (or series of layers) that consists of a sequence of filters. The nature of these filters is trained by the neural network. The image gets convolved by each one of these filters, with the output being stored in an $n$ by n-dimensional "slice" of the next layer.

\section{B. Rectified Linear Unit}

Rectified Linear Unit (ReLU) are used in many CNN architectures as an activation function for the network. In this activation function, the negative co-efficient are replaced with zero value which is represented by the local features of the input image. The function is represented as:

$$
f(x)=\left\{\begin{array}{l}
0 \text { for } x<0 \\
x \text { for } x \geq 0
\end{array}\right\}
$$


Some of the neurons dropped because they do not contribute to forward passage and do not participate in backpropagation. Every time an input is presented, the neural network analyzes another architecture, but all these architectures share a common weight. This technique reduces the complex adaptations of neurons because a neuron cannot rely on the presence of some other neurons.

\section{Pooling Layer}

The pooling layer is simply there to reduce the dimensionality of the previous layer so it is a more appropriate size for the next layer of the network. Typically, this is done with maxpooling, which takes the maximum value of the window the filter is looking at, convolved across the image. A CNN can have any number of convolutional and pooling layers, in any order, with the only limitations being computation power and time, and the risk of overfitting.

\section{Fully-Connected Layer}

The fully connected layer is a regular neural network and is typically used as the final step in a convolutional neural network being used for image classification, where the desired output is an $\mathrm{m}$ element array (with $\mathrm{m}$ being the number of categories of images) containing probabilities of the image being of a particular category.

\section{DEEP LEARNING IN IMAGE FILTERING}

In image filtering applications such as this, both the input and output of the CNN should be an image. Because of this, and for reasons mentioned previously when discussing why traditional neural networks are unsuitable for image processing applications, the fully-connected layer is not needed for networks which are being trained for image filtering.

To gain a better understanding of the ideal network structure and relevant parameters, this report will look at work already done in the field of using deep learning for image filtering, in order to gain some insight into potential ways the final network could be constructed. Seven properties will be investigated:

i. The network structure, in terms of number and size of layers.

ii. Whether a pooling layer is used.

iii. The cost functions.

iv. The activation functions.

v. The evaluation functions.

vi. The number of epochs the network is being trained for

vii. The batch sizes

\section{CONCLUSION}

Image enhancement is an essential preprocessing step in many real time image processing applications. Enhancement of Images is done by many approaches and choice of every approach depends on the type of images. As analyzed, there are various factors which can affect image quality. Some of them are noise, sharpness, distortion, contrast, color accuracy, dynamic range, exposure accuracy, lens flare, etc. These factors must be kept in mind while choosing or designing any image enhancement algorithm. The most common algorithm traditionally used for image enhancement is histogram equalization. Other algorithms were also used but they were somehow an extension or modification of histogram equalization only. As the technology advanced, various new methods were developed for the enhancement of images. As expected, new techniques were better than the traditional image enhancement techniques. As studied from various papers, it has been found that deep learning filters were better than the traditional histogram equalization methods. In future, this review can be used to compare various image enhancement algorithms and their feasibility can also be determined.

\section{REFERENCES}

[1] P. Cunningham, M. Cord and S. J. Delany, "Supervised Learning," in Machine Learning Techniques for Multimedia Case Studies on Organization and Retrieval, Springer, 2008.

[2] G. Cao, L. Huang, H. Tian, X. Huang, Y. Wang and R. Zhi, "Contrast enhancement of brightness distorted images by improved adaptive gamma correction," Computers \& Electrical Engineering, vol. 66, pp. 569-582, 2018.

[3] L. Shen, Z. Yue, F. Feng, Q. Chen, S. Liu and J. Ma, "MSR-net:Lowlight Image Enhancement Using Deep Convolutional Network," ARXIV, 2017.

[4] X. Guo, Y. Li and H. Ling, "LIME: Low-Light Enhancement via Illumination Map Estimation,” IEEE Transactions on Image Processing, vol. 26, no. 2, pp. 982-993, 2016.

[5] X. Guo, "LIME: Low-light IMage Enhancement via Illumination Map Estimation," Google, [Online]. Available: https://sites.google.com/view/xjguo/lime. [Accessed 17 April 2018].

[6] J. Lim, J.-H. Kim, J.-Y. Sim and C.-S. Kim, "Robust contrast enhancement of noisy low-light images: Denoising-enhancementcompletion," in 2015 IEEE International Conference on Image Processing (ICIP), Quebec City, 2015.

[7] Y. Yoo, J. Im and J. Paik, "Low-Light Image Enhancement Using Adaptive Digital Pixel Binning," Sensors - Open Access Journal, pp. 14917-14931, 15 September 2015.

[8] Ramiz, M.A., Quazi, R.: Design of an efficient image enhancement algorithms using hybrid technique. Int. J. Recent Innov. Trends Comput. Commun. 5(6), pp. 710-713, 2017.

[9] E. Schwartz, R. Giryes and A. M. Bronstein, "DeepISP: Learning Endto-End Image Processing Pipeline," 2018.

[10] CS231n, "Convolutional Neural Netowrks (CNNs/ConvNets)," Github, [Online]. Available: http://cs231n.github.io/convolutional-networks/. [Accessed 24 March 2018]. 